14 Ueber den angeblichen Dextringehalt der essharen Kastanien.

\begin{tabular}{|c|c|c|c|c|c|c|}
\hline & 0,50 & $\mathrm{NaO}, \mathrm{SC}$ & $\mathrm{MgO}$, & & $a c$ & \\
\hline I. & 1,3 & 1 & 3,05 & : & 5,7 & : \\
\hline & 2,2 & : & $3, \%$ & : & 6,1 & : \\
\hline
\end{tabular}

Einfache äquivalente Beziebungen zwischen $\mathrm{KO}^{\mathrm{SO} \mathrm{S}^{3}}$ un $\mathrm{NaO}, \mathrm{SO}^{3}$ ergeben sich darans nicht, dagegen stimmen di

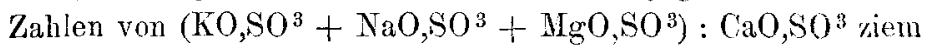
lich überein bei I. wie $5,35: 5,7$,

, II. $, 6,5: 6,1$.

$\mathrm{KO}, \mathrm{SO}^{3}+\mathrm{NaO}, \mathrm{SO}^{3}$ verhält sich zu $\mathrm{MgO}, \mathrm{SO}^{3}$, wie

$\begin{array}{cccc}\text { I. } & 2,3 & : & 3,05 \\ \text { II. } & 3,3 & : & 3,30\end{array}$

Es sind Schwankungen, wie sie von H. Rose sogleicl bei den ersten Untersuchungen der Polyhalite und der Be grïndung der Fornel erhalten wurden und welche immer au die mehrfache Vertretung hinzeigen. In dem vorliegender Falle erstreckt sich diese besonders auf die Alkalien.

Unaufgeklärt ist jedoch das mit Ausnahme der Analyser von II. und V. überall gleichmässig auftretende Resultat dass die Summe der Bestandtheile um 1-2 Procente Ver. luste ergiebt. IV. ist von mir selbst untersucht worden: VI. und VII. von Dreykorn; derselbe hat mehrfache Controle - Versuche eingelegt, so dass die Genanigkeit der Untersuchung eine nur wünschenswerthe ist.

\title{
Ueber den angeblichen Dextringehalt der essbaren Kastanien,
}

von H. Ludwig.

In der Fortsetzung des Handbuchs der organischen Chemie von L. Gmelin, 4. Bd., 1. Abth. S. 631 (hearb. v. Lr. Karl Kraut) findet sich in Betreff des Vorkommens des Dextrins die Angabe: "in den echten Kastanien zu 22,8 bis 23,3 Proc., " nach Albini (Wiener Acad. Ber. 13, 502.). Nach diesen Berichten vom Jahre 1854 enthalten die entschäl- 
Ceber den angeblichen Dextringehalt der essbaren Kastanien.

ten getrockneten Kastanien von verschiedenen Tokalitäten Ttaliens

\begin{tabular}{lr} 
Cellulose & $6,5-8,4$ Proc. \\
Proteinartige Substanzen & $5,2-9,3 "$ \\
Lösliches Pflanzeneiweiss & $0,9-2,1 "$, \\
Amylum & $23,2-38,0 "$, \\
Zucker & $17,5-17,9 "$ \\
Dextrin & $22,8-38,0 "$ \\
Fett & $1,2-2,1 "$ \\
Asche & $3,0-3,3$, \\
\hline
\end{tabular}

Die italienische Abhandlung Albinis: "Ricerche chimiche sul frutto del Castagno" giebt über die Bestimmung des Dextrins nur an: „Per avere la destrina, estrassi i grassi dalle castagne con etere anidro e con alcool assoluto lo $\mathrm{zuc}$ caro; il restante ripetutamente con aqua in cui si scioglieva soltanto la destrina, poichè l'albumina era divenuta insolubile. L'estratto acquoso evaporato e disseccato a $100^{\circ} \mathrm{C}$. veniva pesuto.

Bianche Castagne di Como 23,3 Proc. d. Destrina.

Castagne veronesi 22,8 "

Nach Dessaignes findet sich in den essbaren Kastanien kein Quercit, aber etwas Asparagin. (Journ. pharm. 3. XXV., 25. Liebig-Kopp's Jahresb. f. 1854. S. 666.).

Versuche, welche ich gemeinschaftich mit Herrn Stud. A ug. Burgemeis ter aus Creuzburg, Mitglied meines chem. pharm. Institutes, angestellt habe, um diesen auffällig hohen Gehalt an Dextrin zu controliren, ergaben das Resultat, dass in den hier in Jena im Handel zu erlangenden essbaren Kastanien kein Dextrin vorhanden ist.

Am 23. Februar d. J. wurde von mir eine ungeröstete essbare Kastanie von ihrer braunen Schale befreit, zerschnitten, zerrieben, mit kaltem Wasser angerïhrt und die durch suspendirtes Stärkmehl und Zellgewebe milchige Flüssigkeit auf ein Filter gegeben. Der im Mörser hinterbleibende Gewebsbrei gab mit Jodwasser intensive Bläunng. Das ahgeschlämmte Stärkmehl zeiğte bei 300 facher Linearvergrösse- 
rung theils runde, theils elliptische, theils muschelförmi Stärkekörnchen mit sehr kleiner Centralhöhle. Die Körnche waren kleiner als diejenigen der Kartoffelstärke.

Das klare wässrige Filtrat war fast wasserhell ur nicht merklich gefärbt, röthete nur sehr schwach das blat Lackmuspapier und schmeckte süsslich. Eine Probe de: selben wurde anf Zusatz von bellbraunem Jodwasser dunkle braun, dann hellviolett.

Eisenchlorid färbte eine Probe des wässrigen Auszug hellblau ins Grünliche, eine Portion des auf dem Filte gebliebenen Breies rein blau (eisenbläuender Gerbstoff, vielleicl Gallussäure).

Beim Kochen blieb der Saft klar (also kein Albumin).

Mit Trommers Probe keine Reduction des Kupfer oxyds zu Oxydul. Mit verdünnter Schwefelsäure gekoch dann der Trommer'schen Probe unterworfen, starke Rf duction des $\mathrm{Cu}^{2} \mathrm{O}^{2}$ zu $\mathrm{Cu}^{2} \mathrm{O}$.

Eine Probe des kaltbereiteten wässrigen Auszugs mi der 10 fachen Menge absoluten Alkohols versetzt, gab nu geringe Trübung und nach 24 stündigem Stehen einig Flöckchen, welche gesammelt keine Reduction des $\mathrm{Cu}^{2} \mathrm{O}^{2}$ be der Trommer'schen Probe zeigten.

Hr. A. Burgemeister wiederholte die Untersuchung mi 15 essbaren Kastanien im Anfang März d.J. Von den geschälte: rohen Kastanien wurden 110 Gramme mit kaltem destill. Wasse fein zerrieben, der Auszug durch Leinwand colirt, der Rïckstan nochmals mit Wasser zerrieben, abermals colirt, die Colature vereinigt, durch Filtration daraus das Stärkemehl abgeschie den, abgewaschen und getrocknet. Es betrug 25 Gramme = 22,72 Procent. Das Filtrat wurde im Wasserbade concen trirt, filtrirt und mit dem mehrfachen Volumen starken Wein geists versetzt. Der entstandene Niederschlag wurde au einem Filter gesammelt, nach dem Auswaschen mit Weingeis in Wasser gelöst, mit Thierkohle entfärbt und zur Trocknı verdampft. Es hinterblieben 0,20 Gramme gelblich-weisser mit dunklen Flocken vermischter Substanz, die sich mit Zu rücklassung der Flocken in Weingeist löste. Die Flocker 
waren in Wasser löslich, gaben aber mit Kupfervitriol nebst Natronlauge keine Reduction von Kupferoxydul. Die Gesammtmenge der durch Weingeist gefällten Substanz betrug also nur 0,18 Procent und verhielt sich nicht wie Dextrin. Jedenfalls beruht also die Angabe Albinis auf einem Irrthume.

Dr. A. Bus se, in seiner Abhandlung über das Vorkommen des Dextrins in den Pflanzen (Arch. d. Pharm. 1866. 2 R. 127. Bd. S. 236) fand in den Galläpfeln ebenfalls kein Dextrin, so wie überhaupt kein Gummi. Der durch starken Weingeist fällbare Körper schien gerbsäureartiger Natur zu sein. Er gab mit Jodlösung eine vorübergehend schön violettrothe Färbung.

Eine ähnliche Färbung bemerkte ich (wie oben angegeben) bei dem kaltbereiteten wëssrigen Auszug der essbaren Kastanien. Wir wissen aber, dass Kleister durch Gerbsäure gefällt wird. Es wäre also anzunehmen, dass hier eine Auflösung von Spuren von Amylum neben Gerbsäure vorhanden gewesen sei, welche durch Jodwasser jene vorübergehende violettrothe Färbung bedingte.

Jena, den 16. März 1869.

Kleinere Mittheilungen von Dr. C. Schacht, Apotheker in Berlin.

\section{Ueber Dittmann's Restorative Powder und über ein Pulver ihnlicher Art.}

Vor kurzer Zeit hatte ich Gelegenheit ein dem Ministerium des Cultus etc. eingesandtes Lohpräparat in Pulverform zu untersuchen. Dasselbe verlor bei $100^{\circ} \mathrm{C}$. getrocknet $10,05 \%$ Wasser, gab $1,82 \%$ Asche und enthielt neben viel Pectin 12,5\% Gerbsäure. Letztere wurde nach der von Wagner angegebenen Methode bestimmt, welche nach $\mathrm{C}$. $\mathrm{O}$. Cech und $\mathrm{Ph}$. Büchner die beste aller bis jetzt angegeArch, d. Pharn. CLXXXIX, Bds. 1. u. 2. Hft. 